\title{
Conquiliomorfologia de Tamayoa (Tamayops) banghaasi (Thiele) (Gastropoda, Systrophiidae)
}

\author{
Daniele Pedrosa Monteiro ${ }^{1,2}$ \\ Sonia Barbosa dos Santos ${ }^{1,3}$
}

\begin{abstract}
Conchomorphological aspects of Tamayoa (Tamayops) banghaasi (Thiele) (Gastropoda, Systrophiidae) as a contribution to Systrophiidae systematic. Thirty shells of Tamayoa (Tamayops) banghaasi (Thiele, 1927) from Ilha Grande, Rio de Janeiro, were analyzed with a stereomicroscop showing the following characteristics: discoid shell, without macrosculptures; ovate-lunate aperture without teeth. Thin, sharp and not-reflected lip; umbilicus with $1 / 5$ of the shell's diameter; impressed suture, total whorls from 4 to 5.5 ; protoconch with 1.5 to 2 whorls; yellowish-amber, translucent and glossy periostracum. The morphology of the shells were discussed, as well as the shell's dimensions. The conchomorphological patterns are important for systematic studies of mollusks and this work presents data that can be used in future studies about the family.

KEY WORDS. Systrophiidae, Tamayoa (Tamayops) banghaasi, conchomorphology, taxonomy, Rio de Janeiro
\end{abstract}

THIELE (1931) caracterizou a concha dos moluscos da família Systrophiidae através dos seguintes aspectos morfológicos: concha delgada, na maioria das vezes transparente e brilhante, com textura lisa ou áspera, abóbada (sic) frágil, plana, pouco profunda. Abertura mais ou menos larga, sem dentes. ZILCH (1960) a apresentou como deprimida, transparente, a maior parte das espécies com espira pouco elevada e com as voltas lisas ou, então, com estriações radiais; umbílico mais ou menos largo, com abertura ampla em forma de lua ( sic); margem da abertura simples, cortante, sem dentes. OLIVEIRA et al. (1968), em uma breve classificação conquiliológica dos pulmonados brasileiros, incluiu a família no grupo das conchas disciformes, que se apresentam finas, delicadas, geralmente transparentes, lisas ou ásperas, em geral planas ou fracamente arqueadas. Boca ( sic) com dentes mais ou menos largos ou desdentada. TILLIER (1980) não utilizou os caracteres da concha para diagnosticar a família.

THIELE (1927) descreveu Happia banghaasi, a qual ocorreria nas regiões do Espírito Santo e Olinda [Pernambuco] no Brasil. O autor forneceu uma caracterização geral da concha, descrevendo-a como brilhante, pardacenta, com abóbada ( sic) e voltas frágeis; com quatro voltas e 4,5 mm de diâmetro, $2 \mathrm{~mm}$ de altura e 2,25 mm de abertura.

1) Laboratório de Malacologia, Departamento de Biologia Animal e Vegetal, IBRAG, Universidade do Estado do Rio de Janeiro. Rua São Francisco Xavier 524, 20550-900 Rio de Janeiro, Rio de Janeiro, Brasil.

2) Bolsista PIBIC/UERJ (1997/99). E-mail: danielepm@ hotmail.com

3) E-mail: sbsantos@uerj.br 
Em 1931, o mesmo autor a referiu como Tamayoa banghaasi, no sub-gênero Tamayops Baker, 1928. Caracterizou sua concha como delgada, lisa e brilhante, com a parte superior pouco ressaltada, apresentando 4 a 4,5 voltas, com sutura pouco profunda e abertura larga, em forma de meia lua. Citou sua ocorrência como "Brazil-Trinidad".

O gênero Tamayoa foi criado por BAKER (1925) para Sophina trinitaria Smith, 1898. Na descrição original, incluiu as sub-espécies Tamayoa trinitaria trinitaria (Smith, 1898) e Tamayoa trinitaria venezuelensis. Caracterizou o gênero através da concha e afirmou não ter visto espécimes de "Trinidad" e que teve a precaução de estabelecer o gênero com espécimes nas quais a rádula foi observada. Sobre T. trinitaria venezuelensis chamou atenção para a semelhança com uma Happia Bourguignat, 1889 pequena, mas com sutura larga, porém rasa, impressa e com uma carena no umbílico; na caracterização genérica incluiu também os caracteres radulares.

BAKER (1928) diferenciou o sub-gênero Tamayops, considerando a ausência de carena no umbílico como caracter diagnóstico, e, enfatizando os caracteres radulares como fundamentais para a sistemática dos sistrofídeos. Caracterizou o gênero Tamayoa como portador de um dente raquidiano pequeno, retangular que é tricúspide no sub-gênero Tamayoa e multicúspide no sub-gênero Tamayops. Em ambos os sub-gêneros, o primeiro dente lateral e o segundo têm aproximadamente o mesmo tamanho.

TILLIER (1980) corroborou a diagnose sub-genérica de BAKER (1928), considerando Tamayoa (Tamayoa) como portador de umbílico com carena e Tamayoa (Tamayops) sem carena nesta região.

Desde a descrição e diagnose de THIELE (1927, 1931), a espécie não recebeu nenhum estudo morfológico detalhado. Aparece em alguns trabalhos e catálogos, como HAAS (1939) que a citou para a região da Paraíba; LANGE-DE-MORRETES (1949) que a listou para Espírito Santo e Olinda [Pernambuco] e JAECKEL (1969) que a referiu para áreas costeiras da Bahia até Santa Catarina, partes do Mato Grosso, Paraná e São Paulo.

Os autores apresentam o presente trabalho como subsídio para o conhecimento da concha de Tamayoa (Tamayops) banghaasi, visando permitir futuras comparações da mesma estrutura de espécies congenéricas.

\section{MATERIAL E MÉTODOS}

O material utilizado neste artigo foi coletado entre janeiro de 1997 e dezembro de 1999, em duas áreas de Mata Atlântica, nas proximidades do Centro de Estudos Ambientais e Desenvolvimento Sustentado (CEADS), localizado na Vila Dois Rios, Ilha Grande, Estado do Rio de Janeiro.

Foram estudadas 30 conchas adultas, todas em bom estado de conservação, ou seja, com perióstraco intacto ou pouco desgastado, protoconcha íntegra e teleoconcha íntegra ou com pouco danificada. O material encontra-se depositado na Coleção de Moluscos, Departamento de Biologia Animal e Vegetal, Universidade do Estado do Rio de Janeiro (Col. Mol. UERJ 925-1; 925-2; 942-1; 977-1; 
980-4; 980-5; 990-1; 990-2; 990-3; 1016-6; 1019-1; 1129-1; 1129-2; 1129-3; $1130-1$; 1133-2; 1134-2; 1162-1; 1162-2; 1064-3; 1064-7; 1168-1; 1168-2; 1168-3; $1175-1 ; 1175-2 ; 1241-1 ; 1241-2 ; 1252-2 ; 1313-1)$.

A identificação específica do material foi realizada através da comparação com material identificado pela Dra Norma Campos Salgado, Museu Nacional, Rio de Janeiro, que teve oportunidade de examinar o material depositado na Academy of Natural Sciences of Philadelphia (ANSP). Os síntipos estão depositados no Senckenberg Museum, Frankfurt (SMF 157 994).

A identificação genérica foi confirmada pelo exame da rádula, preparada segundo as técnicas usuais (corrosão em hidróxido de potássio a 5\%, lavagem e montagem entre lâmina e lamínula).

O estudo da forma, da esculturação, dos índices e das relações, obtidas utilizando as dimensões da conchas, permitem a avaliação da conquiliomorfologia e, portanto, esses parâmetros são apresentados e discutidos neste trabalho.

A esculturação compreende sulcos ou ondulações que se destacam, marcando a superfície da concha, apresentando-se distintas das linhas de crescimento (MOORE et al. 1952); neste trabalho são analisadas as macroesculturações, costelas e estrias.

Os índices mostram as dimensões das conchas. São considerados o diâmetro maior (D: limite da borda externa, traçado a partir da borda palatal da abertura até o seu oposto), o diâmetro menor (d: limite entre a borda parietal da abertura e o seu oposto), o diâmetro do umbílico (du: limite entre a sutura da borda parietal externa e o seu oposto), a altura (h: limite entre o ponto mais externo da borda palatal da abertura e a borda do ápice), a largura da abertura (la: limite entre o plano dorso-frontal e o ventro-frontal da borda palatal da abertura), a altura da abertura (ha: limite entre o plano da borda parietal até o ponto oposto mais externo da borda palatal) e o diâmetro da primeira volta (dv: limite entre a sua sutura e o ponto mais externo da volta), de acordo com a metodologia descrita por SOLEM \& CLIMO (1985) e FONSECA (1994), que também foi utilizada para a contagem do número de voltas (Fig. 1).

As relações fornecem instrumentos que permitem inferir sobre a forma da concha, da abertura e do umbílico, além de definir o padrão de crescimento da concha. São elas: h/D -altura da concha dividida pelo seu diâmetro maior (forma da concha); D/du - diâmetro maior dividido pelo diâmetro do umbílico (razão entre o tamanho da concha e o tamanho do umbílico); du/h - diâmetro do umbílico dividido pela abertura da concha (profundidade do umbílico); ha/la - altura da abertura dividida pela largura da abertura (forma da abertura); ha/d - altura da abertura dividida pelo diâmetro menor (forma da abertura) e dv/D - diâmetro da primeira volta dividido pelo diâmetro maior (padrão de aumento do diâmetro da concha) (SOLEM 1976; FONSECA 1994).

As medidas, bem como os desenhos, foram obtidos sob estereomicroscópio Olympus SZH B202 (aumentos de $10 \mathrm{x}$ para as medidas e até 70 x para desenhos e observações da concha) acoplado a câmara lúcida e, quando necessário, com auxílio de paquímetro. A precisão utilizada foi de décimos de milímetro. 


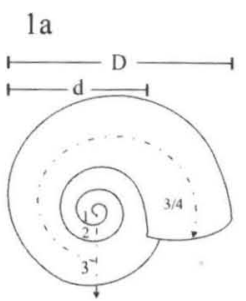

$1 \mathrm{~b}$
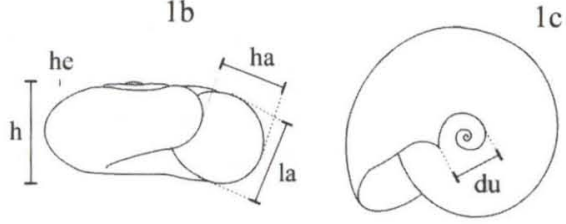

2
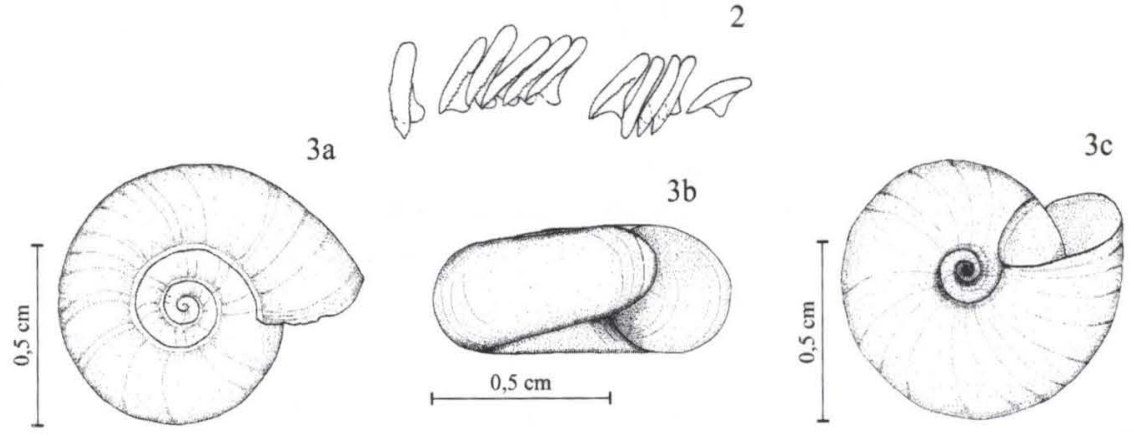

Figs 1-3. (1) Diagramas da concha de Tamayoa (Tamayops) banghaasi: (A) vista dorsal, indicando o modo de contagem das voltas; (B) vista frontal; (C) vista ventral; (D) diâmetro maior; (d) diâmetro menor; (du) diâmetro do umbílico; (h) altura da concha (he) altura da espira; (ha) altura da abertura; (la) largura da abertura. (2) Meia fileira da rádula de Happia banghaasi (Redesenhado de THIELE 1927, sem escala no original). (3) Desenhos da concha de Tamayoa (Tamayops) banghaasi: (A) vista dorsal; (B) vista frontal; (C) vista ventral.

\section{RESULTADOS E DISCUSSÃO}

A espécie em estudo foi identificada como Tamayoa (Tamayops) banghaasi pelo exame da rádula dos exemplares estudados que mostrou a presença de dente central retangular multicúspide com primeiro e segundo laterais de tamanhos semelhantes e denticulados, conforme as caracterizações de BAKER $(1925 ; 1928)$ para o gênero, THIELE (1931) para o sub-gênero e THIELE (1927) para a espécie.

O desenho fornecido por ThiELE (1927) (Fig. 2) para a rádula de Happia banghaasi, representa meia fileira. Aparentemente falta o dente central, mas o primeiro lateral e os cinco dentes seguintes correspondem à rádula dos exemplares analisados neste estudo, os quais apresentam o primeiro dente lateral denticulado de ambos os lados e os cinco laterais seguintes denticulados apenas na face externa.

As conchas de $T$. (T.) banghaasi estudadas apresentam-se conforme a caracterização de THIELE (1931) e ZILCH (1960) para a família, sendo brilhantes, lisas, com abóbada frágil, espira pouco elevada e com abertura larga, de forma arredondada, ligeiramente semilunar (Fig. 3). São discoidais, finas e delicadas, com a abertura sem dentes, de acordo com OLIVEIRA et al. (1968). Têm peristômio fino, cortante e não refletido. A coloração do perióstraco é amarela ambarina, brilhante e translúcida, discordando de ThIELE (1931) e Oliveira et al. (1968) que a 
apresentaram como transparente. Ressaltamos que essa diferença de terminologia pode ser devida a interpretação pessoal dos autores do que seja translúcido ou transparente.

A sutura dos exemplares observados apresenta-se rasa, sem ornamentações como serrilhamentos ou dobras.

BAKER (1925) ao descrever Tamayoa citou que sua sutura é mais larga do que a de Happia e que as conchas adultas são uniformemente arredondadas, concordando com nossas observações.

As conchas apresentam uma pequena elevação da espira, corroborando a descrição de ZILCH (1960) para a família e a de THIELE (1927) para a espécie. Essa elevação foi, em média, $0,02 \mathrm{~cm}$ nos exemplares estudados e não foi mensurada nas descrições anteriores.

As conchas não apresentam macroesculturações, ou seja, não possuem sulcos ou ondulações em sua superfície; não foi observado nenhum tipo de coloração, que pudesse representar manchas ou desenhos que ilustrassem seu perióstraco. Com aumentos superiores a 70 x é possível observar inúmeras linhas de crescimento, distando, em média, $0,01 \mathrm{~cm}$ entre si.

A tabela I apresenta os índices tomados, através de seus valores médios, mínimos, máximos, desvios padrões e variância.

Tabela I. Índices conquiliométricos obtidos de 30 exemplares Tamayoa (Tamayops) banghaasi representados por seus valores médios, máximos, mínimos, desvios padrão e variância. (D) Diâmetro maior, (d) diâmetro menor, (du) diâmetro do umbílico, (dv) diâmetro da primeira volta, (h) altura da concha, (ha) altura da abertura, (he) altura da espira, (la) largura da abertura, (N) número de voltas da teleoconcha, ( $n$ ) número de voltas da protoconcha, (NV) número total de voltas (medidas lineares em centímetros).

\begin{tabular}{lccccccccccc}
\hline & $D$ & $d$ & $d u$ & $h$ & he & la & ha & dv & N & $n$ & NV \\
\hline Média & 0,75 & 0,49 & 0,16 & 0,36 & 0,02 & 0,22 & 0,30 & 0,06 & 3,09 & 1,72 & 4,81 \\
Mínimo & 0,40 & 0,28 & 0,12 & 0,21 & 0,02 & 0,11 & 0,16 & 0,05 & 2,50 & 1,24 & 4,00 \\
Máximo & 0,88 & 0,67 & 0,23 & 0,42 & 0,03 & 0,29 & 0,40 & 0,09 & 4,01 & 2,00 & 5,50 \\
Desvio padrão & 0,10 & 0,08 & 0,03 & 0,04 & 0,00 & 0,03 & 0,04 & 0,01 & 0,29 & 0,19 & 0,32 \\
Variância & 0,01 & 0,01 & 0,00 & 0,00 & 0,00 & 0,00 & 0,00 & 0,00 & 0,08 & 0,04 & 0,10 \\
\hline
\end{tabular}

Os índices médios obtidos mostram-se diferentes daqueles apresentados por THIELE (1927). A média do diâmetro maior da concha é de $0,75 \mathrm{~cm} \pm 0,10$, enquanto o autor citado apresenta um valor de $0,45 \mathrm{~cm}$; a altura da concha foi de $0,36 \pm 0,04$ contra $0,20 \mathrm{~cm}$ apresentados pelo autor. $\mathrm{O}$ número de voltas variou de 4 a 5,5 voltas, com média de 4,8, valor próximo ao apresentado na descrição original, que foi de 4,5 voltas. A média da largura da abertura corroborou a descrição original, sendo igual a $0,22 \mathrm{~cm}$. As variações observadas não representam, a princípio, diferenças significativas uma vez que THIELE (1927) forneceu dados apenas de um exemplar e, não podemos assegurar que seus critérios para obtenção de medidas tenham sido os mesmos utilizados na elaboração deste trabalho.

A tabela II apresenta as relações obtidas, através de seus valores de média, mínimo, máximo, desvio padrão e variância. 
Tabela II. Relações conquiliométricas obtidas de 30 exemplares de Tamayoa (Tamayops) banghaasi representadas por seus valores médios, máximos, mínimos, desvios padrão e variâncias. (D) diâmetro maior, (d) diâmetro menor, (du) diâmetro do umbílico, (dv) diâmetro da primeira volta, (h) altura da concha, (ha) altura da abertura, (la) largura da abertura.

\begin{tabular}{lllllll}
\hline & h/D & D/du & du/h & ha/la & ha/d & dv/D \\
\hline Média & 0,47 & 4,65 & 0,46 & 1,38 & 0,61 & 0,08 \\
Mínimo & 0,43 & 3,33 & 0,34 & 1,20 & 0,50 & 0,06 \\
Máximo & 0,55 & 6,33 & 0,57 & 1,65 & 0,74 & 0,15 \\
Desvio padrăo & 0,03 & 0,53 & 0,05 & 0,12 & 0,06 & 0,02 \\
Variância & 0,00 & 0,28 & 0,00 & 0,01 & 0,00 & 0,00 \\
\hline
\end{tabular}

A média da relação altura/diâmetro maior foi de $0,47 \pm 0,03$, corroborando com a forma achatada da concha, valores maiores do que 1,0 (um) demostrariam uma forma triangular ou mesmo alongada (SOLEM \& CLIMO 1985).

A relação D/du foi de 4,65 , ou seja, o diâmetro do umbílico apresentou-se, em média, com 1/5 do diâmetro maior da concha. Este apresenta-se aberto e profundo. A relação du/h média foi de 0,46 , permitindo a visualização de todas as voltas da concha.

BAKER (1925) sugeriu que o diâmetro do umbílico não ultrapassaria 1/10 do diâmetro maior da concha. Nos exemplares estudados, o diâmetro do umbílico corresponde a $1 / 5$ do diâmetro maior da concha. Esta diferença pode ser específica, uma vez que o autor descreveu o gênero Tamayoa para uma espécie diferente da utilizada neste trabalho.

A abertura apresentou a largura, em média 1,38 vezes maior do que a altura e representou, em média, a metade do diâmetro maior da concha, ressaltando sua forma arredondada, bem aberta.

A relação $\mathrm{dv} / \mathrm{D}$ apresentou valor médio de $0,08 \pm 0,02$, ou seja, a primeira volta representa uma pequena fração do diâmetro maior da concha, sugerindo um crescimento rápido.

\section{CONCLUSÕES}

Considerando os dados disponíveis (descrição e caracterização das conchas, diagnoses do gênero e do sub-gênero e características da rádula de Tamayoa) optou-se por utilizar a combinação taxonômica Tamayoa (Tamayops) banghaasi, conforme apresentada em THIELE (1931).

Os caracteres que identificam a espécie em estudo são: concha discóide sem macroesculturações; abertura arredondada, ligeiramente semilunar, sem dentes; peristômio fino, cortante, não refletido; umbílico com cerca de 1/5 do diâmetro maior; espira pouco elevada, em média $0,02 \mathrm{~cm}$; sutura impressa; perióstraco amarelo ambarino, translúcido e brilhante; número total de voltas variando de 4 a 5,5; número de voltas da protoconcha variando de 1,5 a 2 .

Os dados conquiliométricos apresentados, bem como suas relações, visam auxiliar o estabelecimento de um padrão conquiliológico para a espécie, subsidiando estudos mais aprofundados. Essa caracterização conquiliológica permitirá comparações no âmbito específico e genérico e colabora para a ampliação dos conhecimentos conquiliológicos sobre a família Systrophiidae. 
AGRADECIMENTOS. A Dra Norma Campos Salgado do Museu Nacional do Rio de Janeiro pela identificação específica do material; a UERJ/SR-2 pela concessão da Bolsa de Iniciação Científica no período de 97-99, que permitiu a realização da etapa de campo deste trabalho e ao Setor de Ensino do Curso de pós-graduação da FIOCRUZ, onde este trabalho foi desenvolvido como monografia de especialização em malacologia.

\section{REFERÊNCIAS BIBLIOGRÁFICAS}

BaKer, H.B. 1925. The Mollusca collected by the University of Michigan-Williamson expediton in Venezuela. Occs. pap. Mus. Zool. Univ. Mich. 7 (156): 34-36. 1928. Thiele's Brazilians land snails. Nautilus 41 (4): 124-129.

FonSECA, A.L.M. 1994. Conquiliomorfologia e anatomia dos sistema excretor e reprodutor de Radiodiscus thomei Weyrauch, 1965 (Gastropoda, Stylommatophora, Charopidae). Biociências, Porto Alegre, 2 (1): 163-188.

HAAS, F. 1939. Zur Kentnis der binnen - Mollusken NO Brasiliens. Senckerbergiana 21 (3/4): 254-278.

JAECKEL, S.G.A. 1969. Die mollusken Südamerikas. Monogr. biol. 19: 794- 827.

Lange-de-Morretes, F. 1949. Ensaio de Catálogo dos Moluscos do Brasil. Arch. Mus. Paranaense, Curitiba, 7 (1): 139.

Moore, R.C.; C.G. LALICKer \& A.G. Fischer. 1952. Invertebrates Fossils. New York, McGraw-Hill, $789 \mathrm{p}$.

Oliveira, M.P., E.L. Almeida, I. Vieira \& M.H.R. Oliveira. 1968. Classificação conquiliológica dos pulmonados brasileiros. Bol. Inst. Cienc. Biol. Geoc., Juiz de Fora, 2: 3- 6.

SolEM, A. 1976. Endodontoid land snails from Pacific Islands (Mollusca: Pulmonata: Sigmurethra), part I: Family Endodontidae. Chicago, Field Mus. Nat. Hist., XII+508p.

Solem, A. \& F.M. CLImo. 1985. Structure and habitat correlations of sympatric new Zealand land snail species. Malacologia $26(1-2): 1-30$.

THIELE, J. 1927. Über einige brasilianische landschnecken. Abh. Senckenb. Naturforsch. Ges. 40 (3): 307-328.

- 1931. Handbuch der Systematischen Weichtierkunde. 1. Gastropoda. Stuttgart, Gustav Fisher, 778p.

Tillier, S. 1980. Gastéropodes terrestres et fluviatiles de Guyane Française. Mem. mus. natl. hist. nat., sect. A Zoo., 118: 92-110.

ZILCH, A. 1960. Handbuch der Paläozoologie 6 (2). Berlin, Borntraeger, XII+834p.

Recebido em 10.VIII.2000; aceito em 15.X.2001. 